\title{
The Pitch Control Algorithm of Wind Turbine Based on Fuzzy Control and PID Control
}

\author{
Rui Guo, Jinsong Du, Jinghui Wu, Yiyang Liu \\ Wind Power Control Technology Department, Shenyang Institute of Automation Chinese Academy of Sciences, Shenyang, China \\ Email: guorui@sia.cn
}

Received 2013

\begin{abstract}
Due to the special features of great inertia, pure lag and non-linearity provided with wind turbine, coupled with complex and variable working condition, it is difficult to achieve satisfactory control results simply by employing traditional PID, which has the drawbacks such as adjustment inconvenience, poor anti-interference, and large overshoot, and prolonged adjust span. This paper puts forward one type of improved controller combining Fuzzy Control with PID Control. With larger speed deviation, the controller emphasizes Fuzzy Control to speed system response; with less speed deviation, the controller emphasizes PID Control to improve control accuracy. Simulation Test directing at the algorithm is based on the Bladed software, with the positive result of improved dynamic and static performance of wind turbine under large disturbance.
\end{abstract}

Keywords: Fuzzy Control; Pitch Control; Wind Turbine; Bladed

\section{Introduction}

In general, most current wind turbines, asynchronous doubly-fed or synchronous, adopt pitch control system to ensure safe operation of wind turbines above the rated wind speed and output steady rated power [1]. Pitch control system is considered to have two modes, Hydraulic and Electric pitch-controlled system. No matter driven by any of the two modes, the overall pitch control system is regarded as actuating element in the generator revolving closed-loop control system, implementing pitch angle signal transmitted by the main control system. That is to say wind turbine alters wind-power utilization coefficient by means of changing pitch angle to maintain stable output power [2]. At present pitch controller is simple PID control. Though receiving wide application in industry field, PID control, confined by non-linearity characteristic of wind turbine, makes it difficult to adjust parameters, which leads to untimely restraint of instability caused by external disturbance. The above-mentioned difficulty accordingly brings about a series of problems such as unstable revolving speed, reduced generating efficiency, and quickened wear process, etc. Although some scholars bring forward the theory of applying multi groups of PID parameters on the basis of pitch angle to ensure rapidity and stability of pitch process, it is difficult to tune multi groups of parameters on site [3].

Intelligent control is based upon Control Theory, Information Theory, Artificial Intelligence, Bionics, Neu- rophysiology and Computer Science, and gradually develops to advanced information and control technology. Fuzzy Control is one kind of Intelligent Control. It doesn't need accuracy dynamic model, but uses artificial control rule to organize control decision table, then comes up with outputs.

The paper proposes one type of pitch control algorithm of wind turbine based on Fuzzy Control and PID Control, with flexible and adaptable advantages of Fuzzy Control and accurate characteristic of PID Control. It helps to work out traditional pitch control algorithm, conduct on simulation test platform, and achieves desirable control results.

\section{Asynchronous Doubly-fed Wind Turbine Pitch Control Strategy}

There are two types of Asynchronous doubly-fed wind turbine pitch control strategy based on different rotor speed: above or below rated wind speed area.

In the area below the rated wind speed, the wind turbine is designed to capture wind energy as much as possible, and usually with lower aerodynamic load compared with above rated wind speed area. So the main control system doesn't issue an order to the pitch control system, i.e. the wind turbines run at fixed pitch.

When it is above the rated wind speed, the rotor speed has reached the rated speed area or above. Pitch system should be effective in adjusting wind turbines to absorb 
wind power and reduce the blade load. The main control system is needed to control the pitch control system, thereby changing wind turbine wind-power utilization factor, maintaining the stability of unit output power at rating value.

Pitch control system controls the generator speed, which makes wind turbines stabilize at the rated speed. When the wind speed is relatively stable, pitch controller requires control precision to ensure a smooth output rated power. When the external wind speed increases suddenly, pitch controller should be able to increase the pitch angle in high speed; when the external wind speed suddenly decreased, pitch controller should be able to reduce the pitch angle in high speed. Encountering grid failure cases such as voltage drop causing torque drop, pitch controller should respond to adjustment quickly, not only can increase pitch angle in high speed to ensure no more than the maximum speed, but also avoid off-grid due to decreased generator speed too much.

\section{Design of Fuzzy-PID Pitch Controller}

Wind turbines have the features of great inertia, pure lag, and non-linearity, and with complex and variable working condition, it is difficult to achieve satisfactory control results simply by employing traditional PID, which has the drawbacks such as adjustment inconvenience, poor anti-interference, and large overshoot, and prolonged adjust span. Over the last decade, Fuzzy Control is the product of rapid development of Intelligent Control technology. Because control rules are based on the experience of operator, it can receive desirable control results even without a precise mathematical model. But the nonlinear control decides it has static error.

This paper presents a controller combining Fuzzy Control with PID Control, not only maintains the advantages of PID Control, but also has the characteristics of Fuzzy Control, and introduces a coordination factor used to coordinate Fuzzy Control and PID Control for pitch controller. Control block diagram is shown in "Figure 1".

\subsection{Design of Fuzzy Controller}

According to the actual situation, wind turbine has a rated speed of $1803 \mathrm{rpm}$, taking into account of the maximum speed of $1950 \mathrm{rpm}$, the set allowable deviation is $[-100,+100]$. Selecting the wind turbine revolving speed deviation $e$ and the deviation ratio ee as the fuzzy controller input lingual variables $E, E E$. Selecting pitch angle $u$ as the fuzzy controller output lingual variables $U$ ( $E, E E, U$ are $e, e e, u$ after fuzzy algorithm), thus constituting a dual-input and single-output fuzzy controller. Figure 1 show that the fuzzy controller consists of three function aspects: the fuzzy quantization and fuzzification used for input signal processing, Fuzzy Control algorithm function unit, and fuzzy judgment unit used for defuzzification output.

\subsubsection{Confirm Fuzzy Set and Domain of Input and Output}

Selecting 8 lingual variables to define deviation $E$, mark $E_{1}, E_{2}, \cdots E_{8}$, error range is [-e,e]. Selecting 7 lingual variables to define the wind turbine revolving speed deviation ratio $E E$, mark $E E_{1}, E E_{2}, \cdots E E_{7}$, error range is [-ee, $e e]$. Selecting 7 lingual variables to define output of the Fuzzy Controller, the pitch angle, $U$, mark $U_{1}, U_{2} \ldots$ $U_{7}$.The Fuzzy set is:

$$
\begin{aligned}
& E=\{P B, P M, P S, P O, N O, N S, N M, N B\} \\
& E E=\{P B, P M, P S, O, N S, N M, N B\} \\
& U=\{P B, P M, P S, O, N S, N M, N B\}
\end{aligned}
$$

$P B, P M, P S, P O, O, N O, N S, N M, N B$, represent positive big, positive middle, positive small, positive zero, zero, negative zero, negative small, negative middle, negative big respectively. The Fuzzy domain of $E, E E$ and $U$ as follows:

$$
E=\{-6,-5,-4,-3,-2,-1,-0,+0,+1,+2,+3,+4,+5,+6\}
$$

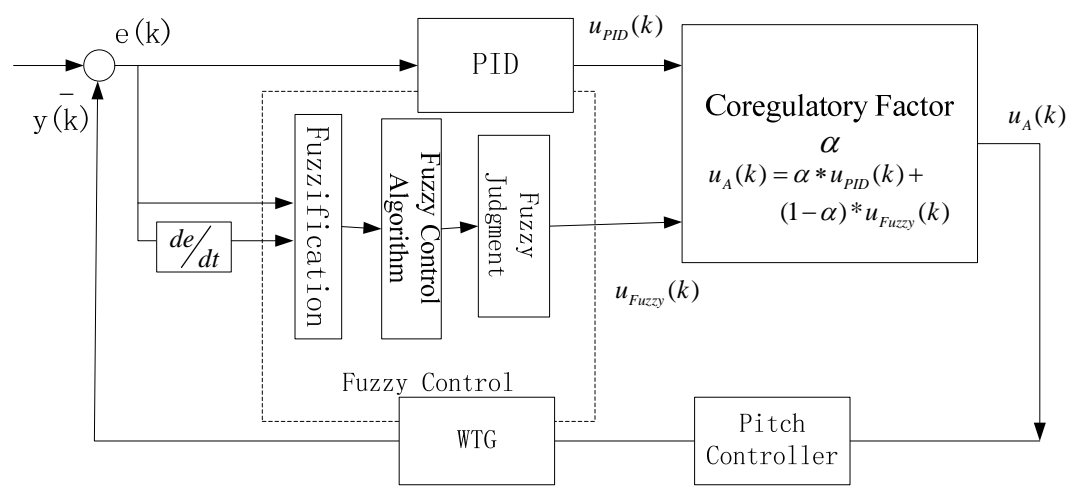

Figure 1. Fuzzy-PID Control Diagram. 


$$
\begin{aligned}
& E E=\{-6,-5,-4,-3,-2,-1,0,+1,+2,+3,+4,+5,+6\} \\
& U=\{-6,-5,-4,-3,-2,-1,0,+1,+2,+3,+4,+5,+6\}
\end{aligned}
$$

The membership is shown in "Tables 1-3".

\subsubsection{Conclude the Control Rules}

Based on the actual work conditions and expertise, after tests again and again, the fuzzy control rules are confirmed as is shown in "Table 4". When the error is negative, and the error ratio is also negative, the error is in an increasing trend. Selecting PB or PM control amount is to remove the existing errors. When the error is negative, and the error ratio is positive, the error is in reducing

Table 1. Membership assignment of $E$.

\begin{tabular}{|c|c|c|c|c|c|c|c|c|c|c|c|c|c|c|}
\hline$E$ & -6 & -5 & -4 & -3 & -2 & -1 & -0 & +0 & +1 & +2 & +3 & +4 & +5 & +6 \\
\hline$P B_{\varepsilon}$ & 0 & 0 & 0 & 0 & 0 & 0 & 0 & 0 & 0 & 0 & 0.1 & 0.4 & 0.8 & 1 \\
\hline$P M_{\varepsilon}$ & 0 & 0 & 0 & 0 & 0 & 0 & 0 & 0 & 0 & 0.2 & 0.7 & 1 & 0.7 & 0.2 \\
\hline$P S_{\varepsilon}$ & 0 & 0 & 0 & 0 & 0 & 0 & 0 & 0.3 & 0.8 & 1 & 0.5 & 0.1 & 0 & 0 \\
\hline$P O_{\varepsilon}$ & 0 & 0 & 0 & 0 & 0 & 0 & 0 & 1 & 0.6 & 0.1 & 0 & 0 & 0 & 0 \\
\hline$N O_{\varepsilon}$ & 0 & 0 & 0 & 0 & 0.1 & 0.6 & 1 & 0 & 0 & 0 & 0 & 0 & 0 & 0 \\
\hline$N S_{\varepsilon}$ & 0 & 0 & 0.1 & 0.5 & 1 & 0.8 & 0.3 & 0 & 0 & 0 & 0 & 0 & 0 & 0 \\
\hline$N M_{\varepsilon}$ & 0.2 & 0.7 & 1 & 0.7 & 0.2 & 0 & 0 & 0 & 0 & 0 & 0 & 0 & 0 & 0 \\
\hline$N B_{\varepsilon}$ & 1 & 0.8 & 0.4 & 0.1 & 0 & 0 & 0 & 0 & 0 & 0 & 0 & 0 & 0 & 0 \\
\hline
\end{tabular}

Table 2. Membership assignment of $E \boldsymbol{E}$.

\begin{tabular}{|l|c|c|c|c|c|c|c|c|c|c|c|c|c|}
\hline$E E$ & -6 & -5 & -4 & -3 & -2 & -1 & 0 & +1 & +2 & +3 & +4 & +5 & +6 \\
\hline$P B_{e \varepsilon}$ & 0 & 0 & 0 & 0 & 0 & 0 & 0 & 0 & 0 & 0.1 & 0.4 & 0.8 & 1 \\
\hline$P M_{e \varepsilon}$ & 0 & 0 & 0 & 0 & 0 & 0 & 0 & 0 & 0.2 & 0.7 & 1 & 0.7 & 0.2 \\
\hline$P S_{e \varepsilon}$ & 0 & 0 & 0 & 0 & 0 & 0 & 0 & 0.9 & 1 & 0.7 & 0.2 & 0 & 0 \\
\hline$O_{e \varepsilon}$ & 0 & 0 & 0 & 0 & 0 & 0 & 0.5 & 1 & 0.5 & 0 & 0 & 0 & 0 \\
\hline$N S_{e \varepsilon}$ & 0 & 0 & 0.2 & 0.7 & 1 & 0.9 & 0 & 0 & 0 & 0 & 0 & 0 & 0 \\
\hline$N M_{e \varepsilon}$ & 0.2 & 0.7 & 1 & 0.7 & 0.2 & 0 & 0 & 0 & 0 & 0 & 0 & 0 & 0 \\
\hline$N B_{e \varepsilon}$ & 1 & 0.8 & 0.4 & 0.1 & 0 & 0 & 0 & 0 & 0 & 0 & 0 & 0 & 0 \\
\hline
\end{tabular}

Table 3. Membership assignment of $\boldsymbol{U}$.

\begin{tabular}{|c|c|c|c|c|c|c|c|c|c|c|c|c|c|c|c|}
\hline$U$ & -7 & -6 & -5 & -4 & -3 & -2 & -1 & 0 & +1 & +2 & +3 & +4 & +5 & +6 & +7 \\
\hline$P B_{u}$ & 0 & 0 & 0 & 0 & 0 & 0 & 0 & 0 & 0 & 0 & 0 & 0.1 & 0.4 & 0.8 & 1 \\
\hline$P M_{u}$ & 0 & 0 & 0 & 0 & 0 & 0 & 0 & 0 & 0 & 0.2 & 0.7 & 1 & 0.7 & 0.2 & 0 \\
\hline$P S_{u}$ & 0 & 0 & 0 & 0 & 0 & 0 & 0 & 0.4 & 1 & 0.8 & 0.4 & 0.1 & 0 & 0 & 0 \\
\hline$O_{u}$ & 0 & 0 & 0 & 0 & 0 & 0 & 0.5 & 1 & 0.5 & 0 & 0 & 0 & 0 & 0 & 0 \\
\hline$N S_{u}$ & 0 & 0 & 0 & 0.1 & 0.4 & 0.8 & 1 & 0.4 & 0 & 0 & 0 & 0 & 0 & 0 & 0 \\
\hline$N M_{u}$ & 0 & 0.2 & 0.7 & 1 & 0.7 & 0.2 & 0 & 0 & 0 & 0 & 0 & 0 & 0 & 0 & 0 \\
\hline$N B_{u}$ & 1 & 0.8 & 0.4 & 0.1 & 0 & 0 & 0 & 0 & 0 & 0 & 0 & 0 & 0 & 0 & 0 \\
\hline
\end{tabular}

Table 4. Fuzzy control rules.

\begin{tabular}{|c|c|c|c|c|c|c|c|}
\hline EC & NB & NM & NS & O & PS & PM & PB \\
\hline NB & PB & PB & PB & PB & PM & PS & O \\
\hline NM & PB & PB & PB & PM & PS & O & O \\
\hline NS & PB & PB & PM & PS & O & NS & NS \\
\hline NO & PM & PS & PS & O & NS & NM & NM \\
\hline PO & PM & PM & PS & O & NS & NS & NM \\
\hline PS & PS & PS & O & NS & NM & NB & NB \\
\hline PM & O & O & NS & NM & NB & NB & NB \\
\hline PB & O & NS & NM & NB & NB & NB & NB \\
\hline
\end{tabular}

trend. It is necessary to take smaller control amount to eliminate the error as soon as possible and prevent the occurrence of overshoot. When the error is positive, and the error ratio is negative, the error is in the decreasing trend. It is advisable to take smaller control amount. When the error is positive, and the error ratio is positive, the error is in increasing trend, selecting the $\mathrm{PB}$ or PM.

Computer language presentation of the above rules are:

$$
\begin{aligned}
& \text { if } \quad \mathrm{E}=\mathrm{NB} \text { and } \mathrm{EE}=\mathrm{NB} \text { then, } \mathrm{U}=\mathrm{PB} \\
& \text { if } \mathrm{E}=\mathrm{NB} \text { and } \mathrm{EE}=\mathrm{NM} \text { then, } \mathrm{U}=\mathrm{PB} \\
& \text { if } \mathrm{E}=\mathrm{NB} \text { and } \mathrm{EE}=\mathrm{NS} \text { then, } \mathrm{U}=\mathrm{PB} \\
& \text { if } \mathrm{E}=\mathrm{NB} \text { and } \mathrm{EE}=\mathrm{O} \text { then, } \mathrm{U}=\mathrm{PB}
\end{aligned}
$$

The control rules can be concluded as follows:

From formula 7 can achieve such fuzzy expression:

$$
R_{1}=\left(N B_{E} \times N B_{E E}\right)^{T} \times P B_{U}
$$

In the same way, the fuzzy relation of $R_{1}, R_{2} \cdots R_{56}$ can be obtained. From the above 56 Fuzzy relation matrix can reach to total Fuzzy relation matrix.

$$
R=\bigcup_{i=1}^{56} R_{i}
$$

Formula 12 represents the overall control decision fuzzy relation matrix, including controller input and output relation. With the relation, the fuzzy amount of output $U$ can get from $E, E E$.

$$
U U=(E \times E E) \circ R
$$

The Assignment is shown in "Table 5".

\subsubsection{Defuzzification}

Applying the maximum membership principle to the output value defuzzification processing in fuzzy control table. Selecting the maximum element $U^{*}$ of membership function as the final output value, $u(k)=U^{*}$. 


\subsection{Confirmation of Coregulatory Factor $\alpha$}

The Confirmation of coregulatory factor $\alpha$ is based on the deviation of speed setting and actual rotor speed feedback. When the deviation is small, the stress is put on PID adjustment, selecting larger value of $\alpha$ to compensate for the Fuzzy Control which can not eliminate the static error; when the deviation is large, emphasis is put on the Fuzzy Control, selecting larger value of $\alpha$ to speed up the system response.

Fuzzy PID controller total output is:

$$
u_{A}(k)=\alpha * u_{P I D}(k)+(1-\alpha) * u_{\text {Fuzzy }}(k)
$$

\section{Simulation Test Platform}

To test the effectiveness of control strategy, the tests are carried out in Simulation Test Platform, adopting the program shown in "Figure 2".

Bladed software is a kind of wind turbine simulation software developed by Garrad Hassan Company, and is the authority of wind turbine design, manufacture and certification. Bladed has been widely used in the international wind power industry, whose software simulation curve has been compared with over 20 series of wind turbine real working curve and prove to be provided with high simulation accuracy[4].

Table 5. Assignment table of the fuzzy control rules.

\begin{tabular}{|c|c|c|c|c|c|c|c|c|c|c|c|c|c|}
\hline EC & -6 & -5 & -4 & -3 & -2 & -1 & 0 & 1 & 2 & 3 & 4 & 5 & 6 \\
\hline-6 & +7 & +7 & +7 & +7 & +7 & +7 & +7 & +4 & +4 & +2 & 0 & 0 & 0 \\
\hline-5 & +7 & +6 & +6 & +6 & +6 & +6 & +6 & +4 & +4 & +2 & 0 & 0 & 0 \\
\hline-4 & +7 & +6 & +7 & +6 & +7 & +7 & +7 & +4 & +4 & +2 & 0 & 0 & 0 \\
\hline-3 & +7 & +6 & +6 & +6 & +6 & +6 & +6 & +3 & +2 & 0 & -1 & -1 & -1 \\
\hline-2 & +4 & +4 & +4 & +5 & +4 & +4 & +4 & +1 & 0 & 0 & -1 & -1 & -1 \\
\hline-1 & +4 & +4 & +4 & +5 & +4 & +4 & +1 & 0 & 0 & 0 & -3 & -2 & -1 \\
\hline-0 & +4 & +4 & +4 & +5 & +1 & +1 & 0 & -1 & -1 & -1 & -4 & -4 & -4 \\
\hline+0 & +4 & +4 & +4 & +5 & +1 & +1 & 0 & -1 & -1 & -1 & -4 & -4 & -4 \\
\hline+1 & +2 & +2 & +2 & +2 & 0 & 0 & -1 & -4 & -4 & -3 & -4 & -4 & -4 \\
\hline+2 & +1 & +1 & +1 & -2 & 0 & -3 & -4 & -4 & -4 & -3 & -4 & -4 & -4 \\
\hline+3 & 0 & 0 & 0 & 0 & -3 & -3 & -6 & -6 & -6 & -6 & -6 & -6 & -6 \\
\hline+4 & 0 & 0 & 0 & -2 & -4 & -4 & -7 & -7 & -7 & -6 & -7 & -6 & -7 \\
\hline+5 & 0 & 0 & 0 & -2 & -4 & -4 & -6 & -6 & -6 & -6 & -6 & -6 & -6 \\
\hline+6 & 0 & 0 & 0 & -2 & -4 & -4 & -7 & -7 & -7 & -7 & -7 & -6 & -7 \\
\hline
\end{tabular}

Table 6. Coordination factor.

\begin{tabular}{|l|l|l|l|l|l|l|l|l|l|l|l|l|l|l|}
\hline$E$ & -6 & -5 & -4 & -3 & -2 & -1 & -0 & +0 & +1 & +2 & +3 & +4 & +5 & +6 \\
\hline$\alpha$ & 0 & 0.3 & 0.5 & 0.8 & 1 & 1 & 1 & 1 & 1 & 1 & 0.8 & 0.5 & 0.3 & 0 \\
\hline
\end{tabular}

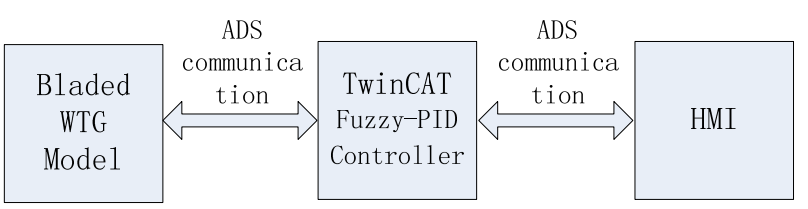

Figure 2. Block diagram of the simulation test platform.
The test platform employs Bladed software for wind turbine model. Through external controller function provided by Bladed software, to write DLL files in accordance with its variable definitions address to achieve data exchange of Bladed and external controller.

Beckhoff PLC offers ADS communication function. Through the ADS functions call, the user can achieve customers-service communications between ADS equipment and PLC tasks to complete the exchange of data between the peripheral unit and PLC.

To achieve real-time data exchange between the Bladed (wind turbine model) and PLC controller (control algorithm), calling DLL file at $20 \mathrm{~ms}$ in Bladed and external controller function block. Through ADS communication reading PLC wind turbine data, HMI monitors the interface and displays the data.

\section{Simulation Result}

Imposing a set of step wind and a set of full-wave wind to simulate wind speed change working condition to verify the rapidity and stability of control algorithm under the circumstance of wind speed change; Simulating low voltage grid failure condition at the $30 \mathrm{~s}$ voltage drop process to verify low voltage ride through (LVRT) performance of control algorithm. HMI interface records the wind speed in the whole process (shown in cyan line), pitch angle (in red line), generator speed (in blue line), torque (in yellow line), power curve (in green line), as shown in "Figures 3-5".

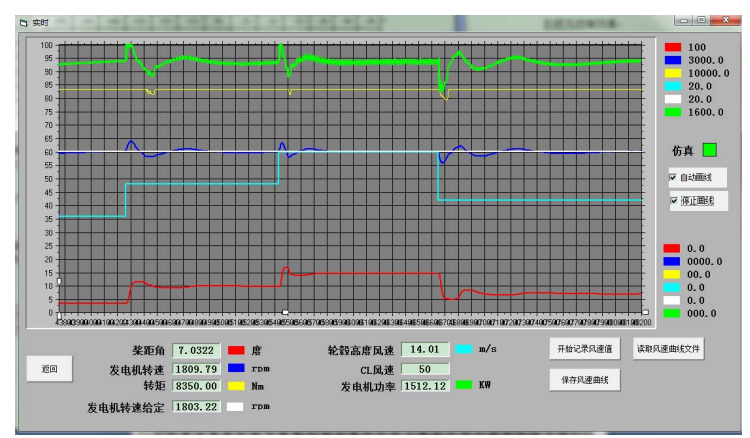

Figure 3. Wind turbine control curve under step wind.

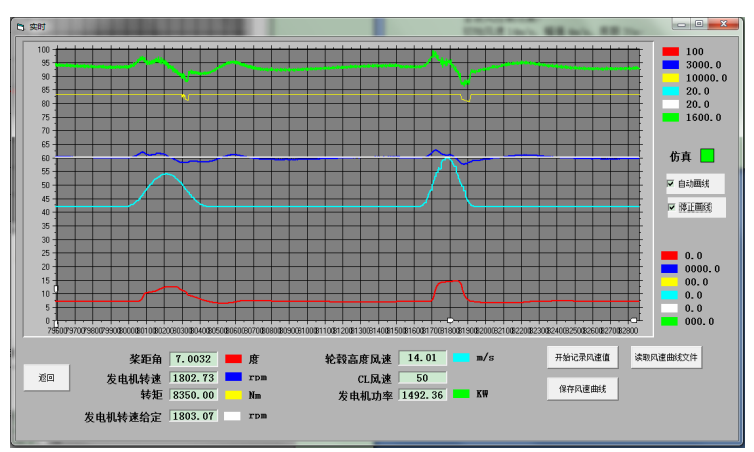

Figure 4. Wind turbine control curve under full-wave wind. 


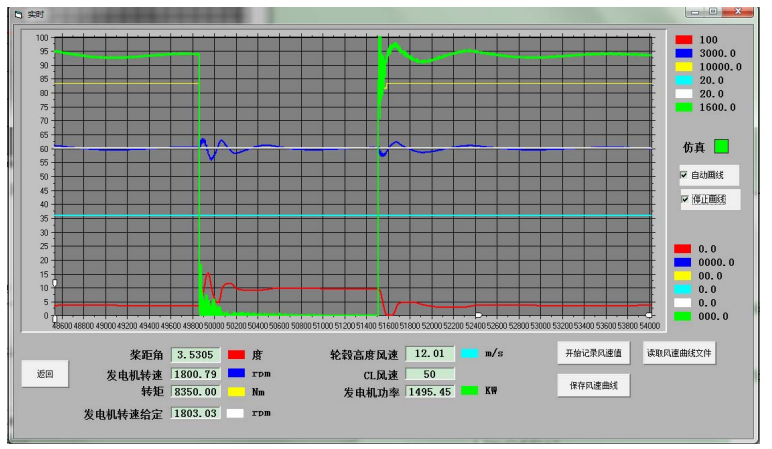

Figure 5. Wind turbine control curve under low voltage fault.

"Figure 3" is control process applying a set of step wind to wind turbine model: (1) wind speed step is from $12 \mathrm{~m} / \mathrm{s}$ to $16 \mathrm{~m} / \mathrm{s}$; (2) wind speed step is from $16 \mathrm{~m} / \mathrm{s}$ to $20 \mathrm{~m} / \mathrm{s}$; (3) wind speed step is from $20 \mathrm{~m} / \mathrm{s}$ step to $14 \mathrm{~m} / \mathrm{s}$.

"Figure 4" is control process imposing a set of full-wave to wind turbine model: (1) Initial wind speed is $14 \mathrm{~m} / \mathrm{s}$, amplitude is $4 \mathrm{~m} / \mathrm{s}$, and period is $30 \mathrm{~s}$; (2) initial wind speed is $14 \mathrm{~m} / \mathrm{s}$, amplitude is $6 \mathrm{~m} / \mathrm{s}$, and period is $25 \mathrm{~s}$.

“Figure 5" is no torque output (low voltage fault) control process applied to the wind turbine model with duration of $30 \mathrm{~s}$. Simply to test the effectiveness of the algorithm, the low voltage fault process is simplified from torque sudden drops to sudden uplift.

Observed from the above simulation curves, with sudden wind speed increase or decrease, the pitch angle can change quickly. When the speed deviation or deviation ratio is relatively great, pitch angle curve slope is large, Fuzzy Control plays a major role, and greatly improves the fast performance of wind turbine; When the speed deviation or deviation ratio is relatively small, the pitch angle slope curve is small, PID Control plays a major role to gradually eliminate static error and improve control accuracy.

Under low voltage fault conditions, the wind turbine torque suddenly drop, the rotor speed increases, the pitch system angle increase quickly to inhibit wind turbine rotor speed from increasing, and gradually stabilize the rotor speed at the rated speed; With failure recovery and torque restore, rotor speed declines. Pitch system angle decrease quickly to restrain rotor speed from decreasing, and gradually stabilize the rotor speed at the rated speed. In this process, wind turbine rotor speed has been well controlled, without speeding or off-grid, and also can be quickly restored to a stable state.

\section{Conclusions}

The paper puts forward an improved pitch control algorithm based upon Fuzzy-PID Control. The method avoids the disadvantages of the traditional simple PID Control such as great inertia, pure lag and non-linearity provided with wind turbine. In the complex and rapidly changing working conditions, the new type controller can effectively improve the static and dynamic performance of the wind turbine; at the same time avoid the wind turbine speeding, and the occurrence off-grid.

\section{REFERENCES}

[1] T. Burton, D. Sharpe and N. Jenkins, "Wind Energy Handbook,” Chichester, UK: John Wiley \& Sons, 2001, pp. 480-492. doi:10.1002/0470846062

[2] H. Y. Ye, "the Control Technology of Wind Turbine Generator, ” China Machine Press, Beijing, China, 2002, pp. 129-133.

[3] B. Wang, Y. Wu, H. Ding, W. M. Yang and W. H. Yu "Variable-pitch Control of Wind Turbine at High Wind Speed," Electric Power Automation Equipment, Vol. 30, No. 8, 2010, pp. 81-83.

[4] T. Y. Luo and B. W. Zhang, "Wind Power Utility Software Products," Shanghai Electric Power, Shanghai, China, Vol. 1, 2007, pp. 103-107.

[5] C. M. Zhang, X. J. Yao, Z. C. Zhang, et al., "A Model Reference Adaptive Blade-pitch Control for A Wind Generator System,” Control Theory \& Application, Vol. 25, No. 1, 2008, pp. 148-159.

[6] X. F. Zhang, D. P. Xu, Y. G. Lu, et al., “Adaptive Fuzzy Control for Large-Scale Variable Speed Wind Turbines,”Journal of System Simulation, Vol. 16, No. 3, 2004, pp. 573-577.

[7] S. Y. Li, "Theory and Application of Fuzzy Control and Intelligent Control,” Harbin Institute of Technology Press, Harbin, China, 1990, pp. 78-123.

[8] W. L. Liu, "TwinCAT PLC Senior programming manual,” Beijing, China, Beckhoff Automation Ltd, 2006, pp. 280-200.

[9] E. A. Bossanyi, “GH Bladed v3.67," Garrad Hassan and Partners Ltd, 2005, pp. 100-107. 